\title{
Dissipative lateral walls are sufficient to trigger convection in vibrated gran- ular gases
}

\author{
Giorgio Pontuale ${ }^{1, \star}$, Andrea Gnoli ${ }^{1, \star \star}$, Francisco Vega Reyes ${ }^{2, \star \star \star}$, and Andrea Puglisi ${ }^{1, \star \star \star \star}$ \\ ${ }^{1}$ Istituto dei Sistemi Complessi-CNR and Dipartimento di Fisica, Università di Roma Sapienza, Piazzale Aldo Moro 2, 00185 \\ Rome, Italy \\ ${ }^{2}$ Departamento de Física and Instituto de Computación Científica Avanzada (ICCAEx), Universidad de Extremadura, 06071 \\ Badajoz, Spain
}

\begin{abstract}
Buoyancy-driven (thermal) convection in dilute granular media, fluidized by a vibrating base, is known to appear without the need of lateral boundaries in a restricted region of parameters (inelasticity, gravity, intensity of energy injection). We have recently discovered a second buoyancy-driven convection effect which occurs at any value of the parameters, provided that the impact of particles with the lateral walls is inelastic (Pontuale et al., Phys. Rev. Lett. 117, 098006 (2016)). It is understood that this novel convection effect is strictly correlated to the existence of perpendicular energy fluxes: a vertical one, induced by both bulk and wall inelasticity, and a horizontal one, induced only by dissipation at the walls. Here we first review those previous results, and then present new experimental and numerical data concerning the variations of box geometry, intensity of energy injection, number of particles and width of the box.
\end{abstract}

\section{Convection in granular media, from slow dense configurations to dilute gas-like setups}

Vibration of granular materials frequently leads to convective patterns [1]. However, the particular mechanism for sustaining and control of a convective dynamics depends upon the particular granular phase under scrutiny [2]. At high packing fraction [3] and low fluidization, "dense convection" has been first identified in the 90's [4-7] and explained through an asymmetric tangential friction at the lateral walls [8-10] or the formation of unstable heaps at the free surface [11]. In granular gases [12-14], on the contrary, the first discovered mechanism is bulk buoyancydriven thermal convection (BBD-TC), initially observed in simulations $[15,16]$. As in molecular liquids $[17,18]$, it is driven by the buoyancy force associated to temperature/density gradients: in BBD-TC such gradients appear spontaneously because of the bulk inelasticity of graingrain collisions [19]. This is a bulk effect: indeed, no particular conditions on the lateral or top boundaries are required for its appearance (for instance in [15] and [20] it appears also with elastic lateral walls) and in fact an analytical study of the stability of the granular gas hydrostatic state without lateral walls (lateral periodic boundary conditions) has shown the emergence of BBD-TC in a region of system's parameters [21, 22]. When lat-

\footnotetext{
${ }_{\star}^{\star}$ e-mail: giorgio.pontuale@ isc.cnr.it

$\star \star$ e-mail: andrea.gnoli@isc.cnr.it

$\star \star \star$ e-mail: fvega@unex.es

$\star \star \star \star$ e-mail: andrea.puglisi@roma1.infn.it
}

eral walls are present (elastic or inelastic) a downward flow velocity is always observed near lateral walls, perhaps because of a reduced buoyancy originated from enhanced dissipation [15, 23]. After the first experimental evidence in 3D [24], other experiments in quasi-2D setups have demonstrated the presence of convection in granular gases [25], but with contradicting features: some of them agree quantitatively with analytical theories where lateral walls are absent [26], while others [27] strongly indicate that convection is almost completely killed when wall inelasticity goes to zero, a scenario - incompatible with BBD-TC theories [21, 22] (see [23] for numerical simulations with similar results).

Recently we have demonstrated the existence of a convection mechanism for granular gases which is alternative to BBD-TC and which necessarily appears in all granular gases fluidized by a vibrating base under gravity, with the only necessary condition being the presence of inelastic lateral walls [28]. Such a mechanism has been called dissipative-lateral-wall-induced thermal convection, "DLW-TC". In this recent study we have employed experiments and simulations to isolate DLW-TC from BBD-TC. In Section 2 we review these recent results, while in Section 3 we present new experimental and numerical data. Conclusions are drawn in Section 4.

\section{DLW-TC}

Let us consider (similarly to the setup sketched in Fig. 1) a 2D low density gas (easily generalized to 3D systems) of identical solid disks of mass $m$ confined by two inelastic 


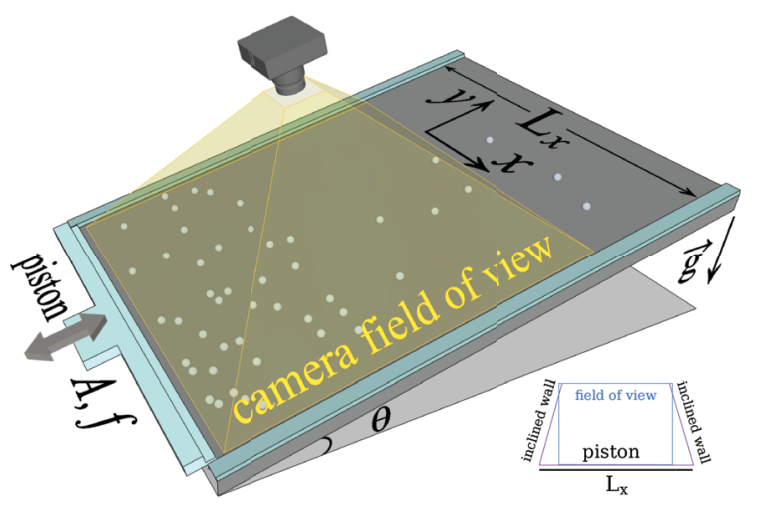

Figure 1. Sketch of the experimental setup. The real length (along $y$ ) of the inclined plane, $L_{y} \gg L_{x}$, is not marked as it is not essential in the description of the system. In the lower-right corner a sketch of the second setup with inclined lateral walls is shown, with a representation (blue square) of the restricted field of view for the fast camera.

parallel walls. The bottom wall provides energy to the system, for instance through steady vibration or (in numerics) in the form of a thermostat. The presence of a top wall or open top boundary does not change our reasoning. A constant gravity field $g$ acts downwards along the vertical $(y)$ direction. In the dilute limit $p=n T$ (with $p$ the pressure, $n$ the number density, and $T$ the granular temperature) [12]. An outgoing energy flux is always originated at a dissipative wall [29], yielding in our case $\partial T / \partial x \neq 0$ at the lateral walls. Momentum balance in the absence of macroscopic flow (hydrostatic) states that

$$
\begin{array}{r}
\partial_{x} p=\partial_{x}(n(x, y) T(x, y))=0 \\
\partial_{y} p=\partial_{y}(n(x, y) T(x, y))=-\operatorname{mgn}(x, y) .
\end{array}
$$

The first equation gives $p(x, y) \equiv p(y)$, which, used in the second equation, sets $n(x, y) \equiv n(y)$ and as a consequence $T(x, y) \equiv T(y)$. This is in contradiction with the horizontal temperature gradient required by dissipation at the lateral walls. Convection must be present.

In [28] we have discussed an experiment (inspired on previous works [30, 31]) under low-gravity conditions (see Fig. 1 and [28], including Supplemental Materials, for details) with $N$ spherical steel beads (diameter $d=1 \mathrm{~mm}$ ) moving inside a cuboid of dimensions $L_{x}=175 \mathrm{~mm}$, $L_{y}=600 \mathrm{~mm}$ and $L_{z}=1.5 \mathrm{~mm}=1.5 d$, thus assuring for a quasi-2D dynamics restricted to the $x y$ plane. A small tilt angle $\theta$ with respect to the horizontal guarantees an effective low value for gravity $g_{\text {eff }} \approx(5 / 7) g \sin (\theta)$, where $g$ is Earth's gravity acceleration [32]. The two lateral "walls" are made of polycarbonate (at $x= \pm L_{x} / 2$ ), characterized by a grain-wall restitution coefficient $\alpha_{w}$, the inferior wall (at $y=0$ ) is a Plexiglas ${ }^{\circledR}$ piston vibrating with amplitude $A$ and frequency $f$, giving an average squared velocity $v_{0}^{2}=(A 2 \pi f)^{2} / 2$. A high speed camera allowed us to reconstruct the average fields $u(x, t), n(x, t)$ and $T(x, t)$. Experiments have been performed in a range of average $2 \mathrm{D}$ packing fraction $\left(v_{2 D}=N \pi(d / 2)^{2} /\left(L_{x} L_{y}\right)\right) v_{2 D} \in[0.1 \%, 1 \%]$, with a constant amplitude of vibration $A=1.85 \mathrm{~mm}$, a range of piston oscillation frequency $f \in[10,45] \mathrm{Hz}$ and a range for the inclination angle $\theta \in[0.011,0.130]$ radiants, leading to a range of rescaled maximum acceleration $\Gamma=A(2 \pi f)^{2} / g_{\text {eff }} \in[70,3800]$. In all our experiments, with the only exception of the lowest number of particles ( $N=100$, where the mean free path is larger than the box's sides) we observed convection with two convective cells that span the full width of the $2 \mathrm{D}$ plane. In all convective cases density and temperature fields display gradients not only in the $y$ but also in the $x$ direction (i.e. transverse to gravity): in particular, temperature decreases moving along the horizontal from the lateral walls toward the center of the system, as expected in view of the outgoing energy flux at the boundaries. Most of the qualitative trends appear similar when $g$ or $N$ are increased, suggesting that these two parameters play an analogous role. In particular the centers of the convective cells move toward the lowest corners (left or right) of the system.

All these features have been confirmed, even in a larger range of parameters, by MD numerical simulations: we have employed an event-driven code for inelastic hard disks. Between collisions the disks perform "free" ballistic fligths under the action of effective gravity $g_{\text {eff }}$. When two disks collide, a normal instantaneous collision with constant restitution coefficient $\alpha \in[0,1]$ is performed. When a disk collides with the base wall, the velocity component parallel to the base is left unaltered, while the normal component is set to a new value extracted from a MaxwellBoltzmann distribution with temperature $v_{0}^{2}$ [33]. When a disk collides with the top wall, an elastic collision is performed. When a disk collides with the lateral walls, an inelastic collision with an infinite mass and with restitution coefficient $\alpha_{w} \in[0,1]$ is performed. Some simulations with the presence of friction along the ballistic flight (introduced by time-discretized dynamics) - which would be analogous to the rolling/sliding friction between spheres and the setup's floor - have also been performed and discussed in [28], giving very similar results. The simulations clearly show that with the parameters of the experiment and the artificial choice of elastic lateral walls the convection is totally suppressed, as expected for experiments at low gravity [21, 22]. In the simulations we have also shown that the intensity of convection decreases linearly with $\alpha_{w}$, in a way similar to the observation of [27] and that the width of the convective cell decreases when $g_{\text {eff }}$ increases. When $g_{\text {eff }} \sim g$ convection is barely visible: this can explain the results and the phase diagram described in [25]. Interestingly, our simulations allowed us to verify that DLW convection is present even when grain-grain collisions are elastic $(\alpha=1)$ provided that $\alpha_{w}<1$ : this suggests that the elastic limit is smooth (a good hint for future theoretical research) and that DLW are sufficient to create not only the horizontal gradient but also the vertical ones, necessary for the developement of convection.

\section{New results}

In Fig. 2 we report the results of two different experiments with the same number of particles $(N=1000)$ and same 

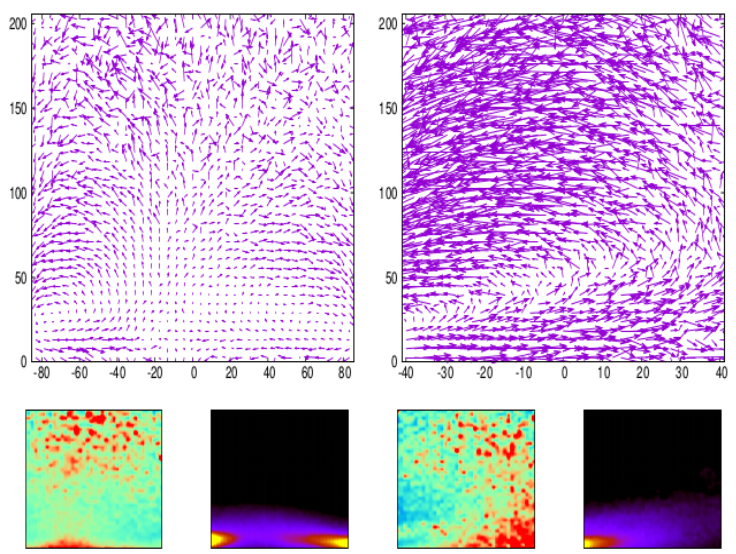

Figure 2. Fields from two experiments with $N=1000, f=45$ $\mathrm{Hz}, A=1.85 \mathrm{~mm}\left(v_{0}=370 \mathrm{~mm} / \mathrm{s}\right)$ and (A): perpendicular lateral walls; (B): lateral walls inclined of 12 degrees s (see sketch in the lower-right corner of Fig. 1). Top row: the velocity field with the two convective cells; coordinates are in units of particle's diameters $(=1 \mathrm{~mm})$. Bottom row: each couple of graphs show the (mass-free) temperature $T / m$ (left) and local packing fraction $v=n \pi(d / 2)^{2}$ (right) corresponding to the case in the top row. The $T$ scale goes from black (colder) at $T=0$ to red (hotter) at $T=T_{\max }$. The $v$ scale goes from black (more dilute) at $v=$ $v_{\min }$ to yellow (denser) at $v=v_{\max }$. Values of $T_{\max } / m v_{0}^{2}$ are: A) $0.08, \mathrm{~B}) 0.15$. Ranges for $\left(v_{\min }, v_{\max }\right)$ are: A) $(0.05 \%, 0.5 \%)$, B) $(0.05 \%, 0.5 \%)$.

driving parameters, but different geometry of the box. The experiment on the left is performed with the usual setup whereas on the right we show a different set-up where the lateral walls form an inwards angle of 12 degrees with respect to the bottom wall direction. Apparently, a small change of inclination of the walls leads to quite an important qualitative change in the velocity, density and temperature field, in particular here we observe only a single convective cell. We stress that we have carefully checked the system to be symmetric with respect to inversion of the $x$ direction, but it is possible that small asymmetries escape our scrutiny. We have not a simple interpretation of this result, and in particular of the symmetry breaking. We expect that within long experiments both clockwise and anti-clockwise convections should be observed, separated by "transition times" which could be very long. We underline that the general mechanism for convection discussed after Eq. (1) does not force the presence of two symmetric cells, it only states that a non-convective steady state is forbidden. In particular, it does not prevent an oscillation between single cells with opposite rotations. In Fig. 3 we report results from different MD simulations with the same number of layers at rest $N \sigma / L_{x}$ (characterising the amount of mass per unit of horizontal length which is the parameters replacing density in an isotropic system such as ours) but different widths of the system, illustrating the remarkable fact that the width of the cell (at fixed layers at rest, gravity and energy driving conditions) is constant and does not depend of the width. This is in perfect agreement with the idea that DLW-TC is a "boundary effect", but nev-
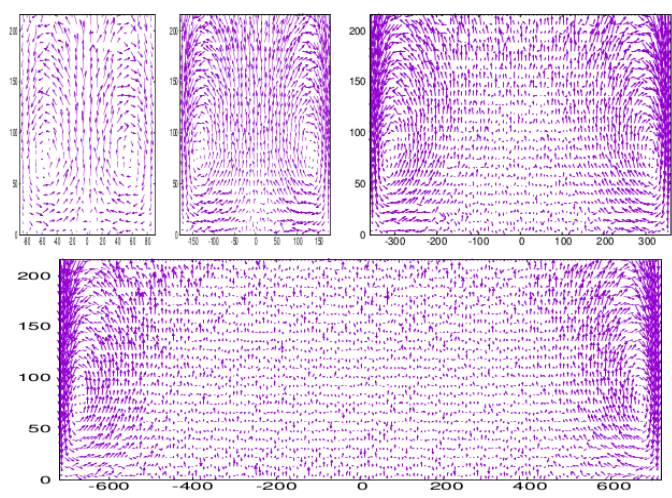

Figure 3. Fields from four simulations with different width and same number of layers at rest $\left(N \sigma / L_{x}=2.78\right)$. From top-left: $L_{x}=180(N=500), L_{x}=360(N=1000), L_{x}=720(N=$ 2000), $L_{x}=1440(N=4000)$.



Figure 4. Width of the convection roll $\left(L_{x}=720\right.$ and $N=2000$ particles) as a function of $v_{0}^{2}$ for different values of $g_{\text {eff }}$.

ertheless it spans a macroscopic portion of the system, i.e. a large number of mean free path (see [28] and its Supplemental Materials for a discussion of Knudsen numbers in our system). In Fig. 4 other numerical results allow us to understand, for several values of $g_{\text {eff }}$, the important role of $v_{0}^{2}$ which represents the intensity of vibration of the base piston, i.e. the unique source of energy in the system. For all values of $g_{\text {eff }}$ the width of the convective cell increase with $v_{0}^{2}$, even if such a growth slows down at high $v_{0}^{2}$. Consistently with previously reported data [28], the width of the cell decreases monotonically with $g_{\text {eff }}$ for low values of $v_{0}$ : on the contrary, it presents non-monotonicy with an initial growth at the first studied values of $g_{\text {eff }}$ followed by a decrease in $g_{e f f}$, for larger values of $v_{0}$.

\section{Conclusions}

In conclusion, we have discussed recent results on a novel thermal (buoyancy-driven) convection effect induced by 
dissipative lateral walls in vibrated granular gases, which at low gravity can be better isolated from the usual convection induced by bulk (grain-grain) inelasticity [28]. In this manuscript we have presented further evidences about the complexity of this phenomenon. In particular we have shown that the inclination of the lateral walls affects the convection in an unexpected way, and we have shown new numerical results with systems of different widths, supporting the idea that DLW-TC is a "boundary-effect", i.e. it is limited to a (macroscopic) region close to the lateral walls. DLW-TC is replaced by usual thermal convection when the parameters are chosen in the region of instability [21, 22], e.g. for high enough bulk inelasticity, as shown in the Supplemental Materials of [28]. We remark that while many studies in dense vibrated granular materials have shown boundary-induced convection, and while other more recent studies in vibrated granular gases have shown the existence of an important role of the lateral wall, the study in [28] is the first to demonstrate the existence of a wall-induced convection in gases which is well distinct from the bulk-inelasticity-induced effect. Such a result is even more fascinating as a theoretical explanation is lacking and cannot be retrieved in the form of a stability analysis (as it was done for bulk thermal convection) because a non-convective simpler base state (either homogeneous or unidimensional [19]) does not exist (i.e., the DLW convective state is the base steady state). This, of course has further implications, for instance, the stability threshold for the BT convection will need to be calculated starting out from the DLWc state and not from a non-convective one.

\section{Acknowledgements}

F. V. R. acknowledges support from Grants FIS201676359-P (Spanish Gov.) and GRU10158 (Junta de Extremadura, partially financed by ERDF funds).

\section{References}

[1] I.S. Aranson, L.S. Tsimring, Rev. Mod. Phys. 78, 641 (2006)

[2] H.M. Jaeger, S.R. Nagel, R.P. Behringer, Reviews of Modern Physics 68, 1259 (1996)

[3] B. Andreotti, Y. Forterre, O. Pouliquen, Granular Media (Cambridge University Press, 20013)

[4] C. Laroche, S. Douady, S. Fauve, J. Phys. 50, 699 (1989)

[5] J.B. Knight, H.M. Jaeger, S.R. Nagel, Phys. Rev. Lett. 70, 3728 (1993)

[6] E.E. Ehrichs, H.M. Jaeger, G.S. Karczmar, J.B. Knight, V. Kuperman, S.R. Nagel, Science 267, 1632 (1995)
[7] J.B. Knight, E.E. Ehrichs, V. Kuperman, J.K. Flint, H.M. Jaeger, S.R. Nagel, Phys. Rev. E 54, 5726 (1996)

[8] J.A.C. Gallas, H.J. Herrmann, S. Sokołowski, Phys. Rev. Lett. 69, 1371 (1992)

[9] J.B. Knight, Phys. Rev. E 55, 6016 (1997)

[10] E.L. Grossman, Phys. Rev. E 56, 3290 (1997)

[11] K.M. Aoki, T. Akiyama, Y. Maki, T. Watanabe, Phys. Rev. E 54, 874 (1996)

[12] J.W. Dufty, Adv. Complex Sys. 4, 397 (2001)

[13] N.V. Brilliantov, T. Pöschel, Kinetic Theory of Granular Gases (Oxford University Press, 2004)

[14] A. Puglisi, Transport and Fluctuations in Granular Fluids (Springer, 2015)

[15] R. Ramírez, D. Risso, P. Cordero, Physical Review Letters 85, 1230 (2000)

[16] P. Sunthar, V. Kumaran, Phys. Rev. E 64, 041303 (2001)

[17] L.D. Landau, E.M. Lifschitz, Mécanique des fluides (Éditions MIR, 1971)

[18] S. Chandrasekhar, Hydrodynamic and Hydromagnetic Stability (Dover, New York, 1981)

[19] F. Vega-Reyes, J.S. Urbach, J. Fluid Mech. 636, 279 (2009)

[20] D. Paolotti, A. Barrat, U.M.B. Marconi, A. Puglisi, Phys. Rev. E 69, 061304 (2004)

[21] X. He, B. Meerson, G. Doolen, Phys. Rev. E 65, 030301(R) (2002)

[22] E. Khain, B. Meerson, Phys. Rev. E 67, 021306 (2003)

[23] J. Talbot, P. Viot, Phys. Rev. Lett. 89, 064301 (2002)

[24] R.D. Wildman, J.M. Huntley, D.J. Parkar, Phys. Rev. Lett. 86, 3304 (2001)

[25] P. Eshuis, K. van der Weele, D. van der Meer, R. Bos, D. Lohse, Phys. Fluids 19, 123301 (2007)

[26] P. Eshuis, K. van der Weele, D. Lohse, D. van der Meer, Phys. Rev. Lett. 104, 248001 (2010)

[27] C.R.K. Windows-Yule, N. Rivas, D.J. Parker, Phys. Rev. Lett. 111, 038001 (2013)

[28] G. Pontuale, A. Gnoli, F. Vega-Reyes, A. Puglisi, Phys. Rev. Lett. 117, 098006 (2016)

[29] P.R. Nott, M. Alam, K. Agrawal, R. Jackson, S. Sundaresan, J. Fluid Mech. 397, 203 (1999)

[30] A. Kudrolli, M. Wolpert, J.P. Gollub, Phys. Rev. Lett. 78, 1383 (1997)

[31] A. Kudrolli, J. Henry, Phys. Rev. E 62, R1489 (2000)

[32] D.L. Blair, A. Kudrolli, Phys. Rev. E 67, 041301 (2003)

[33] F.J. Alexander, A.L. Garcia, Comput. Phys. 11, 588 (1997) 